ACCEPTED MANUSCRIPT

\title{
Action interference in simultaneous and proportional myocontrol: Comparing force- and electromyography
}

To cite this article before publication: Markus Nowak et al $2020 \mathrm{~J}$. Neural Eng. in press https://doi.org/10.1088/1741-2552/ab7b1e

\section{Manuscript version: Accepted Manuscript}

Accepted Manuscript is "the version of the article accepted for publication including all changes made as a result of the peer review process, and which may also include the addition to the article by IOP Publishing of a header, an article ID, a cover sheet and/or an 'Accepted Manuscript' watermark, but excluding any other editing, typesetting or other changes made by IOP Publishing and/or its licensors"

This Accepted Manuscript is @ 2020 IOP Publishing Ltd.

During the embargo period (the 12 month period from the publication of the Version of Record of this article), the Accepted Manuscript is fully protected by copyright and cannot be reused or reposted elsewhere.

As the Version of Record of this article is going to be / has been published on a subscription basis, this Accepted Manuscript is available for reuse under a CC BY-NC-ND 3.0 licence after the 12 month embargo period.

After the embargo period, everyone is permitted to use copy and redistribute this article for non-commercial purposes only, provided that they adhere to all the terms of the licence https://creativecommons.org/licences/by-nc-nd/3.0

Although reasonable endeavours have been taken to obtain all necessary permissions from third parties to include their copyrighted content within this article, their full citation and copyright line may not be present in this Accepted Manuscript version. Before using any content from this article, please refer to the Version of Record on IOPscience once published for full citation and copyright details, as permissions will likely be required. All third party content is fully copyright protected, unless specifically stated otherwise in the figure caption in the Version of Record.

View the article online for updates and enhancements. 


\title{
Action Interference in Simultaneous and Proportional Myocontrol: Comparing Force- and Electromyography
}

\author{
Markus Nowak ${ }^{1}$, Thomas Eiband ${ }^{1}$, Eduardo Ruiz Ramírez ${ }^{2}$ and \\ Claudio Castellini ${ }^{1}$ \\ ${ }^{1}$ Institute of Robotics and Mechatronics, DLR - German Aerospace Center, \\ Wessling, Germany \\ ${ }^{2}$ SDU Robotics, The Maersk Mc-Kinney Moller Institute, University of Southern \\ Denmark \\ E-mail: markus.nowak@dlr.de
}

February, 2020

\begin{abstract}
.
Myocontrol, that is, control of a prosthesis via muscle signals, is still a surprisingly hard problem. Recent research indicates that surface electromyography (sEMG), the traditional technique used to detect a subject's intent, could proficiently be replaced, or conjoined with, other techniques (multi-modal myocontrol), with the aim to improve both on dexterity and reliability. In this paper we present an online assessment of multimodal sEMG and force myography (FMG) targeted at hand and wrist myocontrol. Twenty sEMG and FMG sensors in total were used to enforce simultaneous and proportional control of hand opening/closing, wrist pronation/supination and wrist flexion/extension of 12 intact subjects. We found that FMG yields in general a better performance than sEMG, and that the main drawback of the sEMG array we used is not the inability to perform a desired action, but rather action interference, that is, the undesired concurrent activation of another action. FMG, on the other hand, causes less interference.
\end{abstract}

Keywords: myocontrol, surface electromyography, force myography, prosthetics, target achievement control, action interference

Submitted to: J. Neural Eng.

\section{Introduction}

Smooth, natural control of upper-limb prostheses (an instance of myocontrol) is the typical problem which looks simple from an abstract point of view and turns out to be extremely hard in practice. Back in the Fifties surface electromyography (sEMG), 


\section{Online combination of FMG and sEMG}

formerly a musculoskeletal condition diagnostic technique, began to be used in a two-sensors configuration to open and close a one-degree-of-freedom (DOF) motorized gripper - actually, the first self-powered hand prosthesis in history. Surprisingly, this rudimentary form of control is, still today, unsurpassed in practice, although (multisensor) sEMG was targeted by control theorists and mathematicians soon after the pioneers' era (an early example can be found in [11]).

Yet, dexterous myocontrol, e.g., control of multi-DOF self-powered prosthetic hands and wrists, is still by and large unsolved, the main problem being unreliability in daily-life activities. On top of this, upper-limb prosthetic hardware is still expensive, heavy and clumsy: these are the main reasons why self-powered prostheses are so often rejected $[12,23]$, although better functionality and control are highly desired characteristics in the population of patients $[6,8]$. Only two commercially available solutions employing machine learning are known, namely Complete control by COAPT Engineerings and the Myo Plus by Ottobock. Proper myocontrol is a surprisingly hard problem and fifty years of research have not yet produced a reliable, dexterous, natural and clinically accepted system, enabling upper-limb amputees to smoothly control their prostheses [2].

Specifically, if we consider the human-machine interface devoted to enforcing myocontrol, multi-modal sensing is one of the solutions the community is attempting $[9,12,15]$. The idea is to gather more information from the surface of the amputee's missing limb than sEMG currently can, by using different kinds of sensors as a substitute of, or as a companion to, sEMG. Novel sensor modalities are being explored, which could yield information less prone to the well-known problems of sEMG (sweat, muscle fatigue, variability of the signal during isometric contractions due to motor unit recruitment); as well, they should be targeted at gathering information which sEMG cannot in principle provide such as, e.g., the status of deep muscles [3].

In this paper we focus upon one such alternative technique, force myography (FMG). As opposed to sEMG, which directly detects the electrical fields generated by muscle contractions, FMG uses pressure sensors placed on a body part of interest to interpret the deformations induced on the stump by said contractions. While contracting, muscles bulge and change the shape of, for instance, the forearm, in ways that can be quite reliably be associated to the actions enforced by the human wrist and hand [24]. FMG has potential to detect different information with respect to sEMG [26], provides similar accuracy and better-conditioned signals than sEMG [5,32] and has already been tested offline and online even on amputees [4]; but as far as we know, studies on the combination of FMG and sEMG while in action are still scarce (a remarkable example being, e.g., [1]).

Building on our own previous work, in this paper we report about an experiment in which several intact subjects were fitted with twenty sEMG and FMG sensors on the forearm; they were then engaged in a repetitive online goal-reaching task involving the opening/closing of the hand, flexion/extension and pronation/supination of the wrist an instance of the Target Achievement Control (TAC) test [28]. It is worthwhile to stress 


\section{Online combination of FMG and sEMG}

that the task was online, although still in controlled conditions (i.e., not in a daily-livingactivity setup), since offline performance can only offer limited information of online performance in myocontrol $[14,21,30]$. The results of our experiment indicate that for proportional control, particularly for fine movements, requiring low forces, sEMG does not suffice and is outperformed by FMG. The shortcoming can be traced back to the unintended activation of an action, when trying to perform a different action, a phenomenon we call action interference.

\subsection{Related work}

Surface EMG $[16,17]$ detects a superposition of many Motor Unit Activation Potentials, filtered by the tissue the signal travels through. These signals are, electrical fields generated by motor units during muscle contraction. FMG [24,31], on the other side, detects the pressure exerted by the muscles towards the surface of the skin by volumetric changes induced during muscle activity. Due to the/very different nature of the signals gathered by these two techniques, it seems reasonable that they could be proficiently fused in order to better detect a subject's intent.

In particular, FMG alone has already been tested by and large, and has proved to yield a signal which is more resilient to motion artefacts and fatigue than sEMG [32], and has been directly applied to amputees: in [4] four amputated subjects were able to enforce six primary grips through classification, with an accuracy of above $70 \%$. On the other hand, FMG and sEMG have been/comparatively examined but in parallel, i.e., without combining them, in $[5,26]$. The results shown therein indicate that FMG provides a signal which is less oscillatory during isometric contractions than sEMG, thereby providing a significantly better performance during intent detection, performed using a regression approach, i.e., without classification of patterns but rather enforcing simultaneous and proportional control.

To the best of our knowledge, this study represents the first attempt to mix sEMG and FMG in an online task, with the aim of determining how to best combine the two techniques. In our own previous work [20], an offline analysis was performed of data obtained in conditions similar to the ones we report about here. The results therein showed that (a) it is not important how the sensors are laid out on the forearm, but (b) it can make a significant difference how the signals are combined. In particular, four ML approaches were tested, and it was determined that sEMG alone performed significantly worse than any other approach (i.e., FMG alone or combined with sEMG in two different ways). Moreover, quite surprisingly, it was determined that the best way of combining the two techniques consisted of just feeding to the ML system the sEMG and FMG signals juxtaposed. This approach led to smaller normalised root-mean-squared error in the offline analysis, as well as to better success ratio and shorter task completion times in a preliminary online test, performed on one subject only. This very work can be therefore viewed as the natural companion and completion of the above-mentioned paper. 


\section{Online combination of FMG and sEMG}

\section{Materials and Methods}

As an extension of [20], where the comparison was performed mostly offline (only a single user online test), this study involved 12 able-bodied subjects in a completely online goal-reaching scenario - an instance of the Target Achievement Control test [28]. Furthermore, the design of this study allows an in-depth comparison of the approaches for different types of goals that the subjects had to reach. These goals differ as far as the action (the hand gesture) that had to be performed is concerned, as well as regarding the level of activation, the intensity / force to which the action had to be performed. An example would be wrist flexion at level 0.33. Here the subjects would need to flex their wrist to $33 \%$ of full flexion to reach the goal $\neq$ Notice that, in this work, we have intentionally left out the problem of predicting combined actions (e.g., grasping while pronating the wrist) since it would have led to too complex an experimental protocol, and it would probably have failed due to the small number of sensors.

\subsection{Participants}

We engaged 12 able-bodied subjects in our study (three women, nine men; age between 22 and 45; all but one right handed). Prior to the experiment all participants received written and oral descriptions of the experiment. After all questions about the experiments and associated risks were answered, all participants signed an informed consent form. This study was formally approved by the host institution's internal committee for data protection and it followed the guidelines of the World Medical Association's declaration of Helsinki.

\subsection{Experimental Setup}

The participant was comfortably seated in front of a computer screen and asked to wear a sEMG and FMG acquisition device that consists of two separate bracelets. A depiction of the full setup and the bracelet can be found in Figure 1.

The ten sEMG and ten FMG sensors were arranged in alternating order on the bracelets to cover the full circumference of the forearm by both the sEMG and FMG sensors. The influence of different sensors arrangements has already been investigated in [20] and no significant influence of the different sensor selections has been found. Therefore, we were not required to change the bracelet placement and sensor organisation for each of the four configurations we intended to compare.

On the screen the participants were shown two hand models, see the left image of Figure 1. Each of these hands serves a particular purpose. The left/grey hand serves as a stimulus to the participant. During the data acquisition or ML training, it indicates to the user which hand action to perform. During the goal-reaching part of the experiment, it indicates the target hand position the participant has to reach. The right/beige hand

† The percentage is related to a level set by the subject. The experimenter asked the subject to perform a tense, but comfortable level of force. 

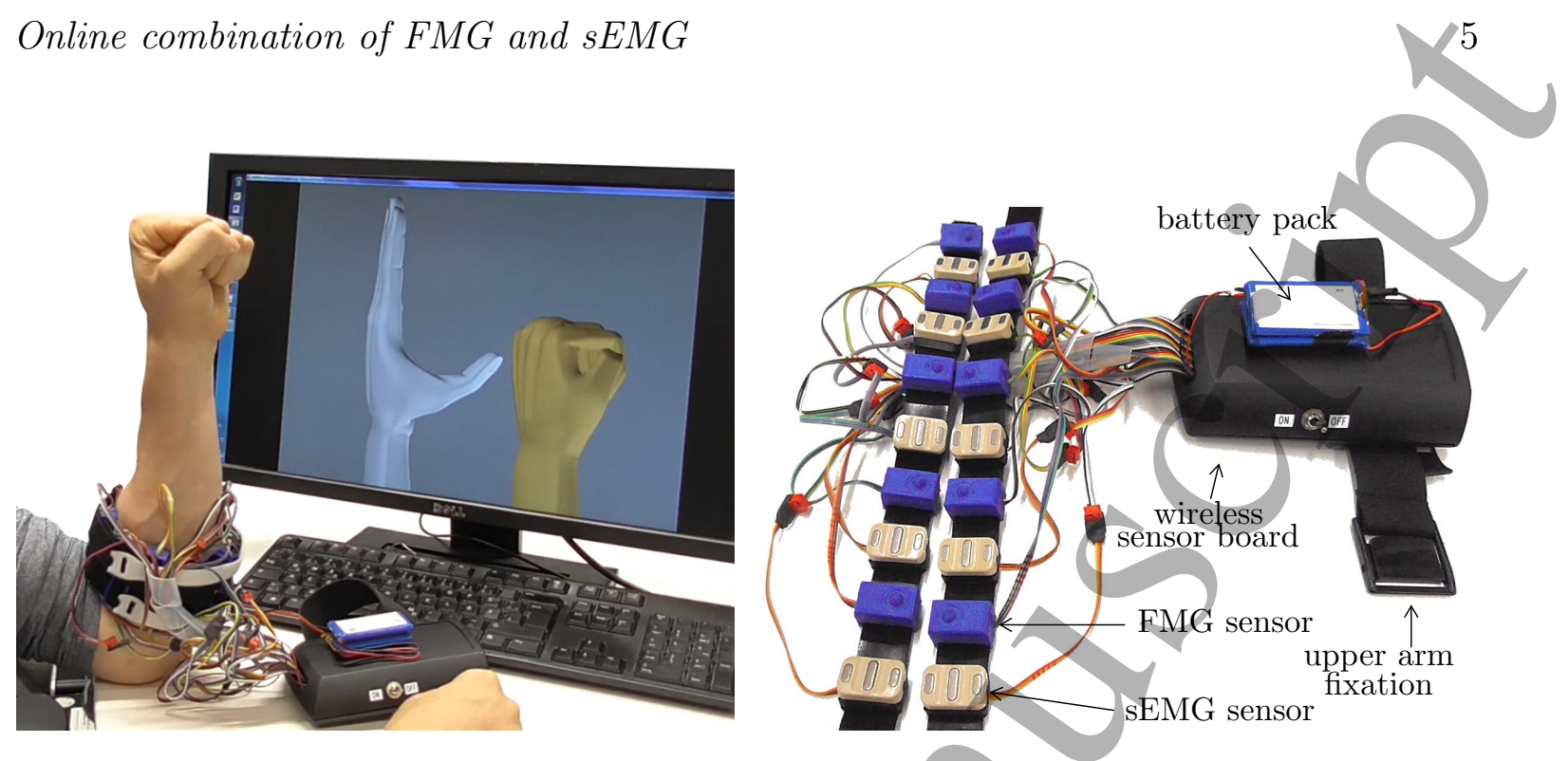

Figure 1: (left) the forearm of a participant wearing the sensors, the wireless data acquisition device, and the computer screen showing a target hand configuration (grey hand) and the current prediction of the ML algorithm (beige hand). (right) The wireless data acquisition device, consisting of a battery powered analog-digital converted and two bracelets with sEMG and FMG sensors.

is controlled by the user. It displays the prediction of the ML configuration that is currently in use. It is only active in the goal-reaching part of the experiment. With this setup the goal reaching becomes a matching task of left and right hand.

\subsection{Hardware and Signal Processing}

The sEMG electrodes are of type Ottobock 13E200=50 Myobock with internal filtering electronics, supplying an amplified, rectified and band-pass filtered sEMG signal. This sensor type has been designed for clinical applications. Explicit details about internal electronics and filters are not publicly available. The FMG sensors and its electronics are custom made and described in more detail in [5]. Basically, voltage over a force-sensing resistor (FSR) is amplified and then digitized. The FSR is embedded in a flexible 3Dprinted housing. The sampling rate of both sEMG and FMG sensors is $100 \mathrm{~Hz}$. Signals of both types have been filtered on the software side, using a 1st-order Butterworth low-pass filter with a cut-off frequency of $1 \mathrm{~Hz}$. The output after the filtering stage is directly used for training and prediction.

\subsection{Experimental Protocol}

The experiment consists of two major parts. First, labelled muscle activation data is gathered from the participant, which is used to train a ML algorithm in four different signal mixing configurations. The underlying ML method is always the same. The difference lies in the sensors selection as well as in the modality of mixing the two sensor types. Furthermore, depending on what sensor type is used a hyperparameter is varied. 


\section{Online combination of FMG and sEMG}

The four ML configurations are the same as in the offline analysis performed in [20], therefore the hyperparameters have been taken from that analysis.

The ML algorithm that we used is the well established Ridge Regression with Random Fourier Features (RRRFF), first introduced in prosthetic control/in [13] and successfully used by this group numerous times $[18,19,22,29]$. This algorithm can be seen as a finite dimensional approximation of a Support Vector Machine (SVM) using a Radial Basis Function Kernel. This algorithm has certain in our opinion highly important characteristics, e.g. bounded in space and therefore fast computation, incrementality, and proportionality.

The central point of this work is an in-depth comparison between sEMG, FMG and the mixture of both. For this purpose we compared four configurations of training our ML algorithm, based on i.e. sEMG only, FMG only, a stacked mixture of both (STA) and a hierarchical mixture of both (ENS). The hierarchical configuration will be referred to as ensemble learning [7].

For the RRRFF algorithm, there is a target signal for each of the actions to be trained. These target values represent the hand/wrist configuration for the specific action. For the ENS model, a set of target signals is obtained from each, sEMG and FMG models. These target values correspond to the target values from the sEMG and FMG models, which are the predicted finger/hand configurations. Then a third model is trained with the stacked output of the first two models. From that third model, the final target values are obtained.

A visualisation of all four mixing configurations can be found in Figure 2.
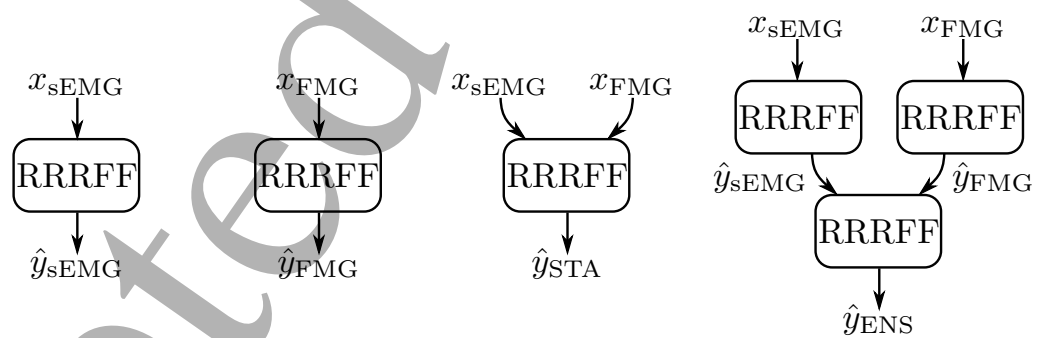

Figure 2: Visual representation of the four different ML configurations investigated in this work. From left to right: sEMG only, FMG only, stacked mixing (STA) and ensemble learning (ENS). This is a supplement to the description in Section 2.4.

To guarantee a fair comparison between these four configurations we always used a subset of ten sensors to test each configuration. This means we used all ten of the respective sensors, when we trained the ML algorithm for one specific sensor type only, but reduced the number of each sensor type to five, when training a mixing approach. Following this chain of thought, we were able to acquire training data once from all 20 sensors and train all four ML configurations with a subset of this data. This effectively reduced the duration of the experiment, easing the participants' task. Following the stimulus (grey hand model) the participant had to perform six different hand and wrist actions, namely rest or relaxed (no action), power grasp, wrist flexion, wrist extension, 
Online combination of FMG and $s E M G$

wrist pronation and wrist supination. A depiction of these actions can be found in Figure 3 .
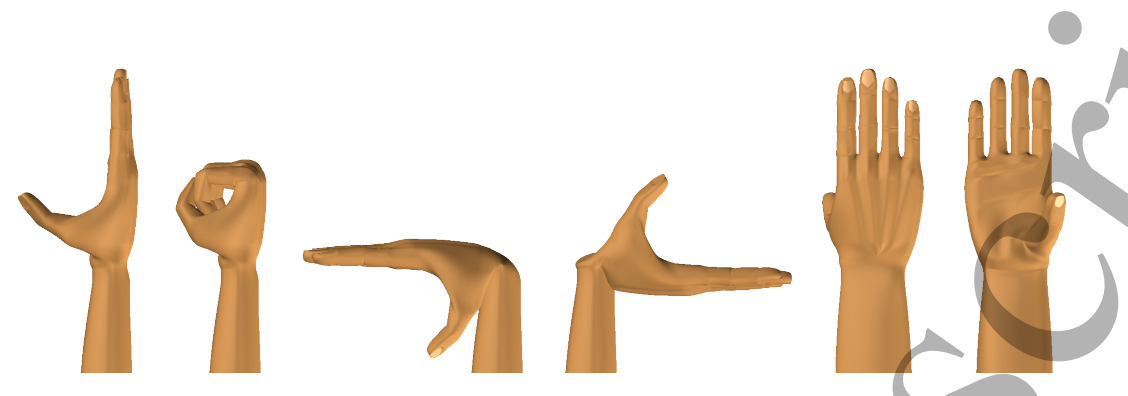

Figure 3: Depiction of the six actions the participants had to perform during the ML training phase.

These six actions were repeated 5 times. After the data acquisition and the subsequent ML training the participant was allowed to quickly test the quality of the online prediction performing free movements and comparing them to the predicted actions. In case the participants verbally stated that they are not satisfied the training session was repeated until the participants were satisfied with the performance. Thereafter the participants were presented with 120 goal reaching tasks. This segment of the experiment lasted on average $29^{\prime} 50^{\prime \prime} \pm 3^{\prime} 20^{\prime \prime}$. The distinctive feature of these tasks is the fact that we train only on "on/off"-data, i.e. full activation of a particular action, but the goals can be intermediate values for these actions (a realistic training method already defined in [27]). Figure 4 depicts different levels for the actions wrist flexion and wrist extension. No updates or retraining were allowed once the first goal was presented to the participants.

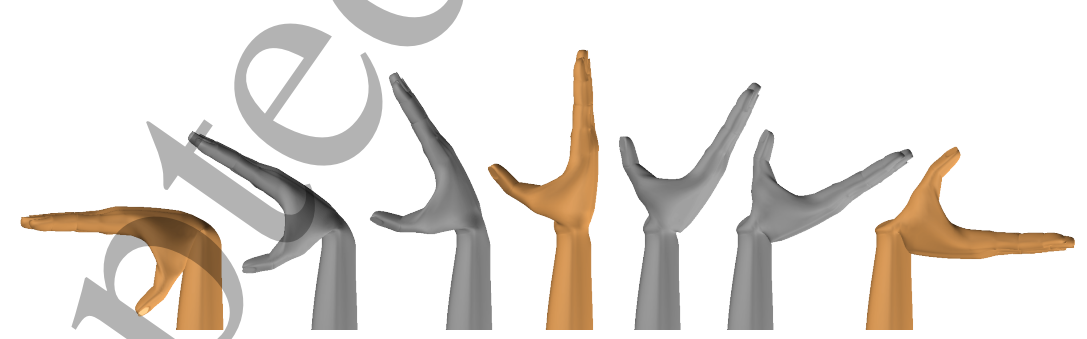

Figure 4: Range of targets using the example of wrist flexion, rest and wrist extension. The levels from left to right refer to full (1.0) wrist flexion, 0.67 wrist flexion, 0.33 wrist flexion, rest, 0.33 wrist extension, 0.67 wrist extension, full (1.0) wrist extension. For clarification: For ML training we only used the beige actions, while the participant were asked to match all of the target configurations depicted here.

In the training phase the user only performed the first (full wrist flexion), the middle (rest) and the last (full wrist extension) action. While in the goal reaching phase the user was asked not only to reach those full activations, but intermediate levels of these activations as well, i.e. at a level of 0.33 and 0.67 . For each of the four ML configurations we asked the user to perform two repetitions of these five actions at three different levels 


\section{Online combination of FMG and sEMG}

(excluding relaxed/no action). Hence, we end up with 120 tasks. To assure that time dependent effects, e.g. a learning effect or fatigue, have a limited influence on our results we presented each subject with a different order of the three factors we were varying, i.e. the ML configuration, the action and the level.

The measure to evaluate the performance of each ML configuration is the success or failure in reaching each individual goal. For each task the participant had $15 \mathrm{~s}$ to finish the task. Successfully finish means reaching the target area (approx. $1.2 \%$ of the work space) and staying in that area for $1.5 \mathrm{~s}$. Once the target area is left the timer resets. The design of the study allows us to compare the different mixing configurations at different action levels and for different actions.

\section{Experimental Results}

Since successfully reaching a goal or not is a binary outcome measure, we used a loglinear analysis [10] to investigate the outcome of our study. A visualisation of the fitted model using a mosaic plot can be found in Figure 5

The Figure is split into four major columns and three major rows, representing the four ML configurations and the three levels of activation. Furthermore, in each major column there are five minor ones, which represent the five different actions, and in each major row there are two minor ones, which represent the relative relation between successful and failed tasks for each task type $\S$.

Two more characteristics are highlighted in this plot. First, the borders of each block are either solid or dashed. A solid line represents a positive deviation from the expected value, while a dashed line represents a negative deviation from the expected value. Second, while the majority of blocks are grey some are coloured in teal or purple. A teal block represents a positive deviation from the expected value as well, but in this case the deviation is significant. Purple blocks represent a significant negative deviation. The colour is based on the Pearson residuals, which is a version of a standardised residual. Values $>2$ or $<-2$ imply that the deviation from the expected value is significant.

Remarkably, the number of cases with a significant deviation increases as the level of activation decreases: in three cases the number of failures is significantly higher than expected whereas in six cases the number of failures is significantly lower than expected. The three cases with significantly more failures all occur when the ML algorithm is trained only on sEMG data and at lower levels of activation of the degree of freedom (DOF) of wrist flexion/extension. For these three cases we have plotted the absolute error per DOF for the failed tasks in Figure 6. Additionally, for each case we performed a one-way ANOVA to compare the error of the three DOFs. The results are the following:

- $F(2,57)=7.027, p=0.002$ for wrist extension at level 0.33

$\S$ A task type is determined by the action performed, the ML configuration used and the level of activation. 
Online combination of FMG and sEMG

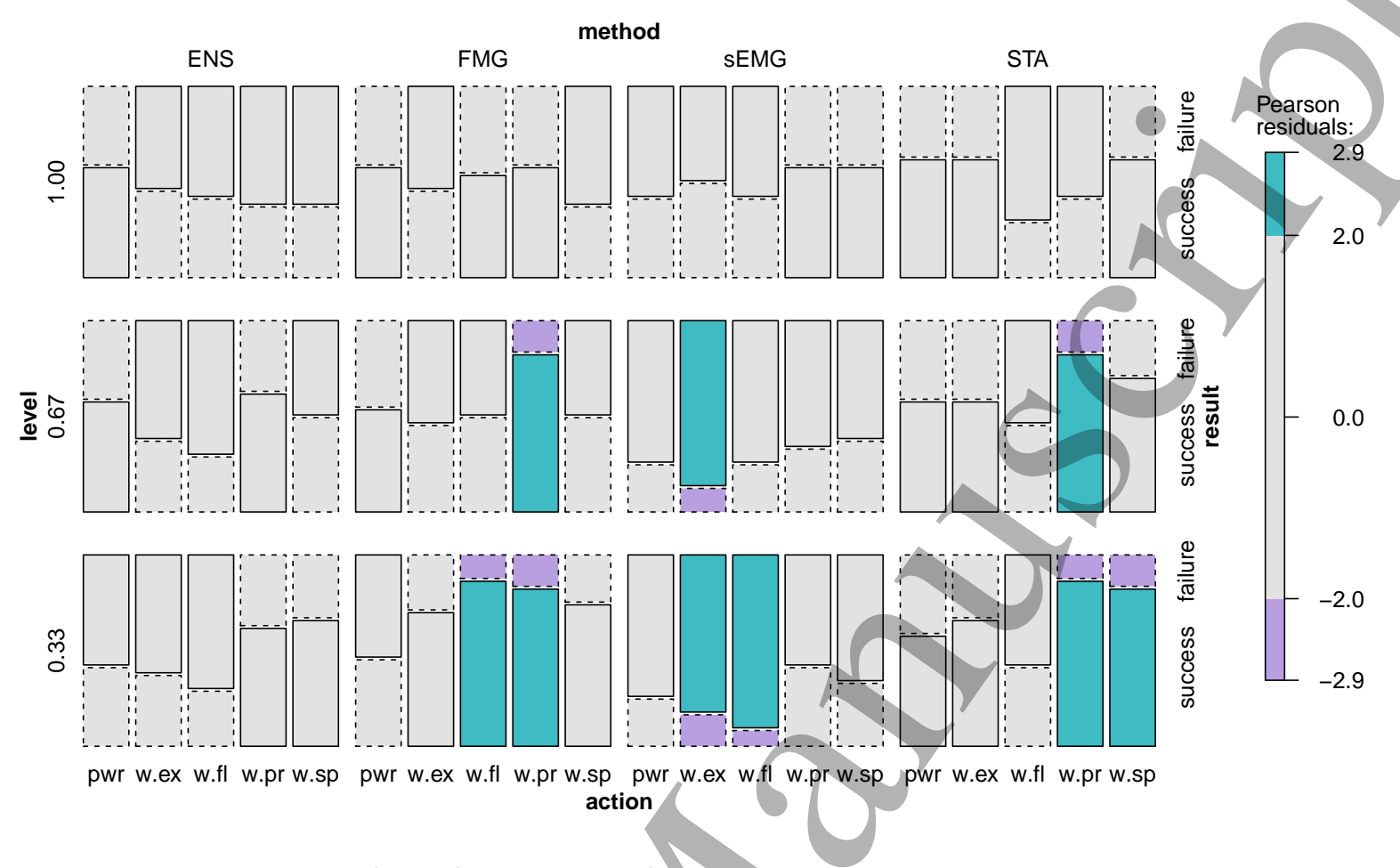

Figure 5: Mosaic plot of the full results of the experiment. Major columns represent the different ML configurations, minor columns represent the different actions (pwr: power grasp, w.ex: wrist extension, w.fl: wrist extension, w.pr: wrist pronation, w.sp: wrist supination), major rows represent different level of activation and minor rows show the relative relation between successful and failed tasks. Dashed outlines imply a negative deviation from the expected value, while solid outlines imply a positive deviation. Grey blocks mean that a deviation is not significant, while teal stands for a significant positive deviation and purple stands for significant negative deviation.

- $F(2,63)=4.002, p=0.02$ for wrist flexion at level 0.33

- $F(2,60)=4.070, p=0.02$ for wrist extension at level 0.67

Since significant difference was found we followed up the one-way ANOVA with a Tukey Test. The results can be found in Table 1.

For Figure 5 we investígate the saturated model of the log-linear regression. We chose this test, since the result of each task (success or failure) is binominal and we only preformed two repetitions of each task per subject to reduce the duration of the experiment. Therefore, we are not able to analyse the success rate in a sensible way without reducing the data along one of the factors. However, this information would provide a broader understanding of the results of the experiment. Hence, we preformed said reduction along each of the three factors, which we depict in three boxplots in Figure 7 .

Furthermore, we highlight the difference between the four ML configurations with three additional plots at each activation level. For this purpose we have collapsed the two factors "action" and "configuration" into one factor and compared this new factor 
Online combination of FMG and sEMG
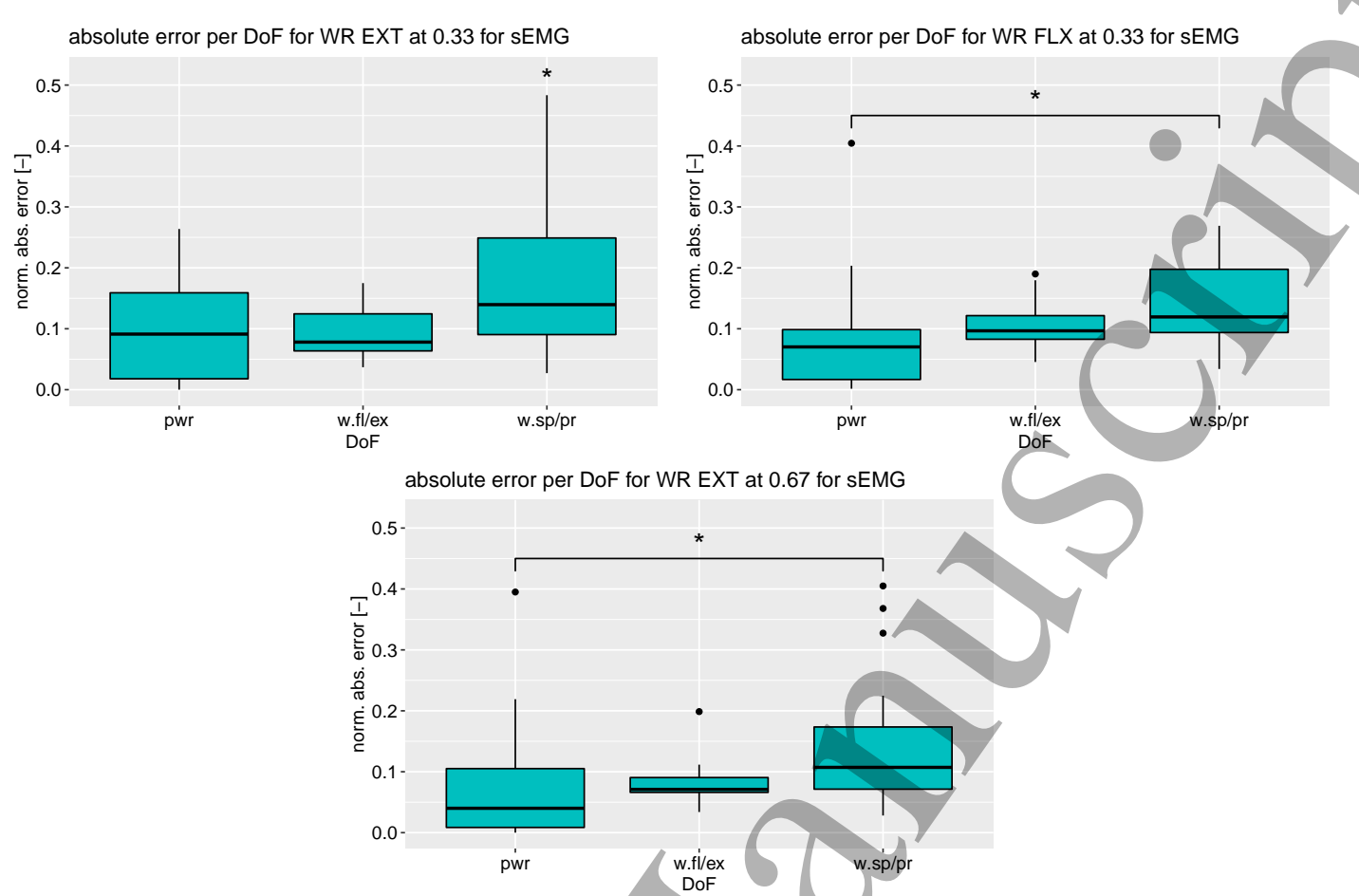

Figure 6: Boxplot of absolute error per DOF for failed tasks for the three cases, where the number of failures is significantly higher than expected. Brackets with an asterisk imply significant difference. A group with an asterisk implies significant difference from all other groups.

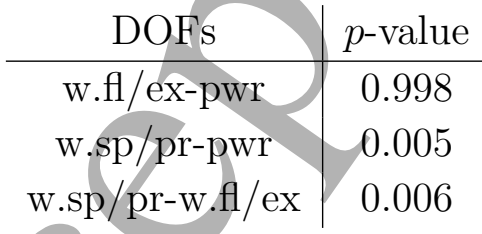

(a) for wrist extension at level 0.33

\begin{tabular}{c|c} 
DOFs & $p$-value \\
\hline w.fl/ex-pwr & 0.521 \\
w.sp/pr-pwr & 0.018 \\
w.sp/pr-w.fl/ex & 0.209
\end{tabular}

(b) for wrist flexion at level 0.33

\begin{tabular}{c|c} 
DOFs & $p$-value \\
\hline w.fl/ex-pwr & 0.955 \\
w.sp/pr-pwr & 0.031 \\
w.sp/pr-w.fl/ex & 0.061
\end{tabular}

(c) for wrist extension at level 0.67

Table 1: Results of post-hoc Tukey-Test for the cases plotted in Figure 6. 
1

2

3

4

5

6

7

8

9

\section{Online combination of FMG and sEMG}
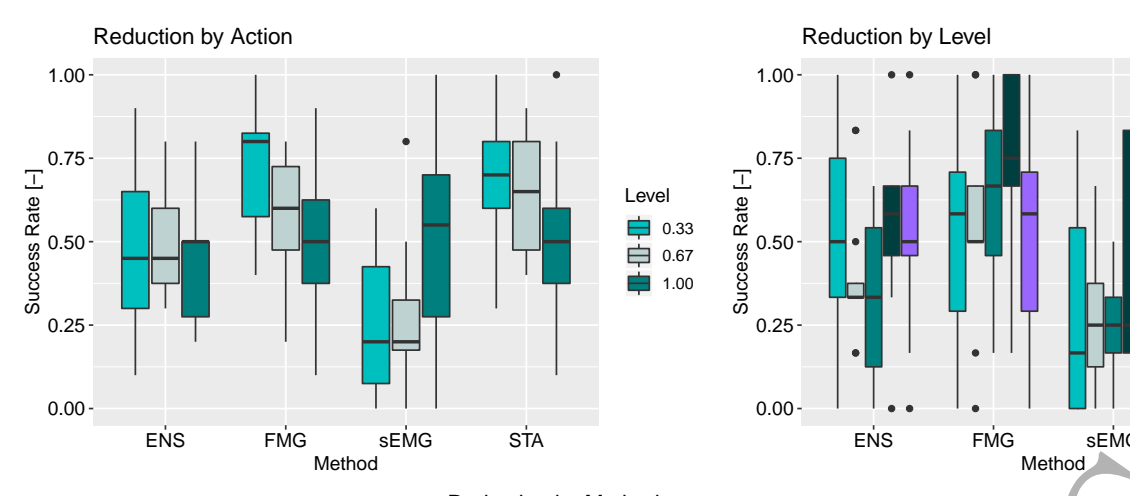

11
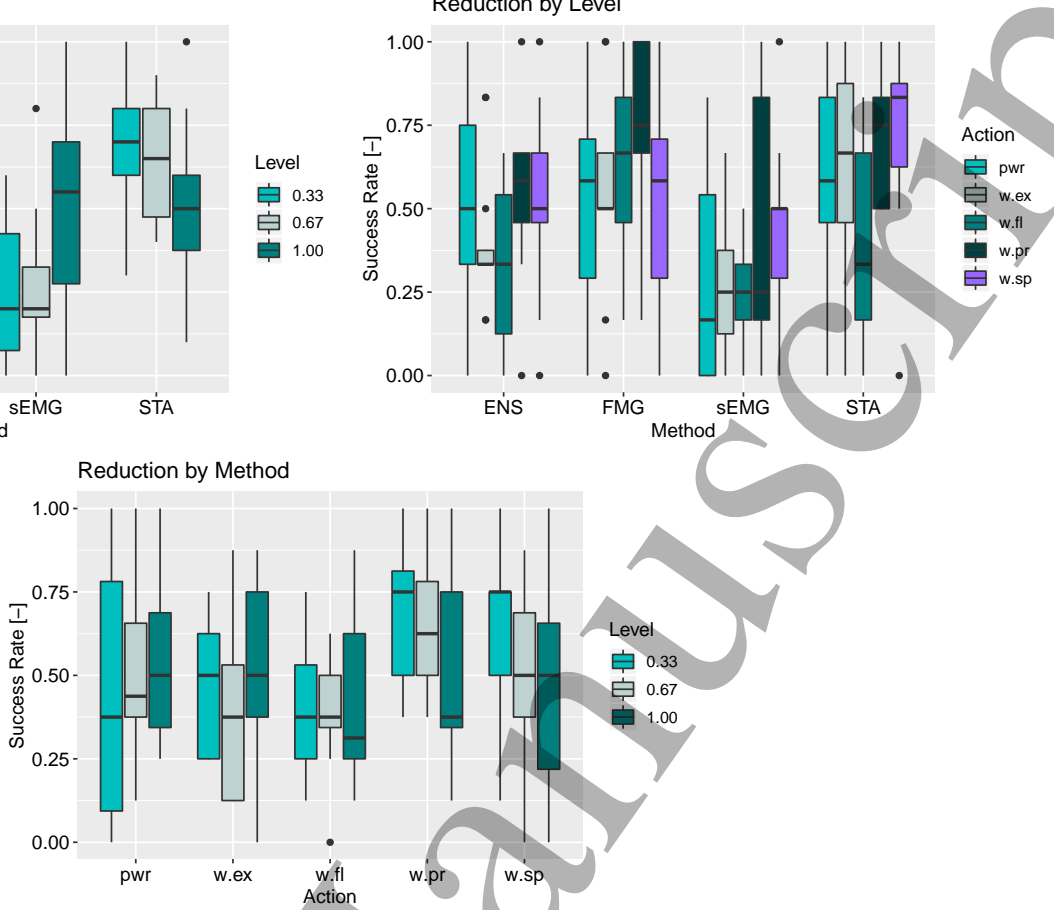

官 0.67

두 1.00
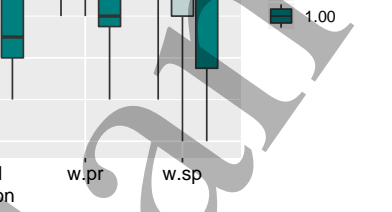

Figure 7: Boxplot of reduction by each of the three factors. The success rate is calculated or each subject and the boxplots visualise the success rate across all 12 subjects.

by the number of successful tasks (Figure 8). To some extent this is a rearrangement of Figure 5 by success rate over all subjects and repetitions.

\section{Discussion and conclusions}

Can FMG be proficiently coupled with sEMG for simultaneous and proportional myocontrol, and if so, how? The experimental results we obtained let us draw two major conclusions. Firstly, there are statistically significant differences in performance, according to the different sensor type set and mixing approach; secondly, the difference becomes larger at lower levels of activation.

Regarding the first issue, in general, the configurations involving FMG perform better than those involving sEMG, and by simply "stacking" FMG and sEMG sensors together we get better results than by using the more complicated ensemble mixing. When FMG alone or the stacked approach are used, we get significantly better performance for the lower activation levels of the wrist - especially for wrist pronation. The ensemble learning seems to somehow "mix" the signals in such a way to cancel out the poor performance of sEMG, but the stacked approach additionally preserves the good performances of FMG. So FMG seems, all in all, to perform better than sEMG, both alone and when combined with it.

As far as the second issue is concerned, this fact is not surprising: during lowactivation tasks the magnitude of both sEMG and FMG signals is accordingly low, 
Online combination of FMG and $s E M G$
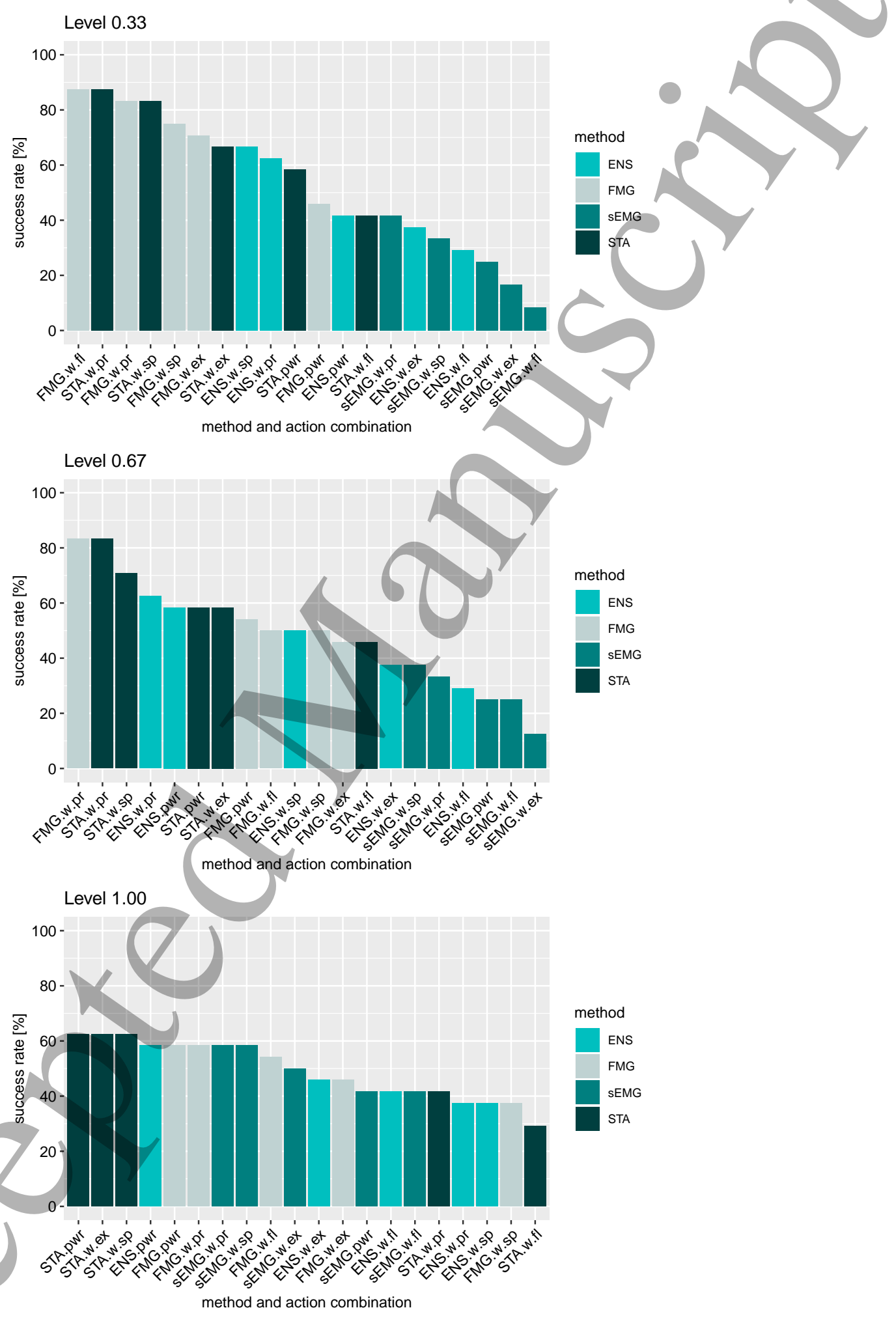

Figure 8: Number of successful tasks for each action and method combination sorted in descending order for activation level 0.33 (top), 0.67 (middle) and 1.0 (bottom). Colours highlight the ML configuration that was used. 


\section{Online combination of FMG and sEMG}

therefore the related signal-to-noise ratio decreases, quite obviously leading to worse performances. Furthermore, at full activation level (1.00) all ML configurations perform comparably well, whereas at the lower levels differences become more evident. It is here worthwhile to stress that all ML configurations were trained only on data provided at level 1.00, hence we claim that FMG generalises better than sEMG across activation levels. Actually, the sEMG-alone approach performs significantly worse than expected - these cases occur for wrist flexion and extension at the lower levels of activation.

\subsection{Action interference: a major reason of failure}

It is interesting to have a deeper look at the reason behind these failures. We therefore compared the absolute error obtained for each DOF while performing an action - that is, not restraining the analysis to the error obtained for the DOF required for that specific action, see Figure 6. This was done to determine whether, in general, the goals could not be reached because the desired DOF could not be activated to the desired level, or because another DOF was being simultaneously unintentionally activated. All subplots of Figure 6 indicate that the source of failure was an inadvertently high wrist pronation or supination, never the inability to flex or extend the wrist. This phenomenon, which we call action interference, has already been observed [25] and seems to be related to the sEMG signal while it is basically absent in the FMG signal.

Figure 9 visualises this phenomenon. Using linear discriminant analysis (LDA) as a dimensionality reduction technique we created a 3D representation of the training data of one single subject. We selected the subject with the overall success rate closest to the overall median. We can clearly see that for sEMG data some actions, i.e. wrist flexion (light teal) and wrist pronation (purple), are clustered close to the rest action. Assuming an approximately linear increase in sEMG activation, one would need to "pass through" an action that is close to the rest action, when trying to reach an action that is far from the rest action. Therefore, activating an action far from the rest cluster at a low level inevitably leads to a coactivation of the action close to the rest action. This behaviour is what we call action interference. On the other hand, in case of FMG the action clusters seem to be evenly spread around the rest action, which would explain why action interference appears to be absent in this case. Here we would like to refer to the supplementary material, where the interested reader can find a rotating version of these 3D plots.

Although we cannot yet exactly say why this is the case, we speculate that it could be due to the location of the muscles involved in the actions we tested. As a matter of fact, while the muscles used to flex / extend the wrist are superficial, the muscles involved in pronation and supination are deep, inducing a relatively smaller magnitude of the sEMG signal due to the connective and fat tissue it has to travel through. As opposed to that, FMG records the superficial deformation of the forearm occurring when pronating / supinating, which has in principle nothing to do with the location of the musscles, but rather with the global bulging induced by the muscle activation. 


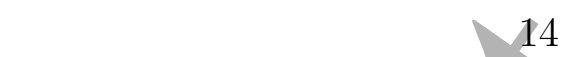

SEMG

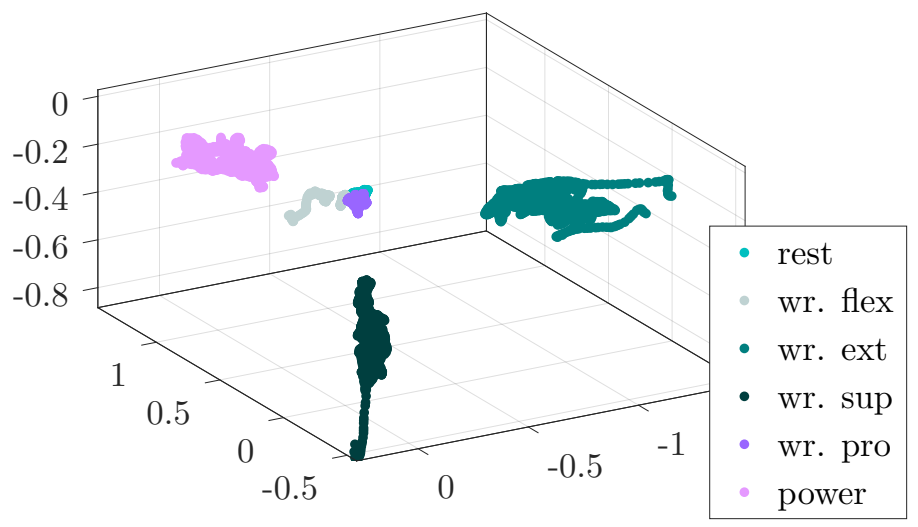

FMG

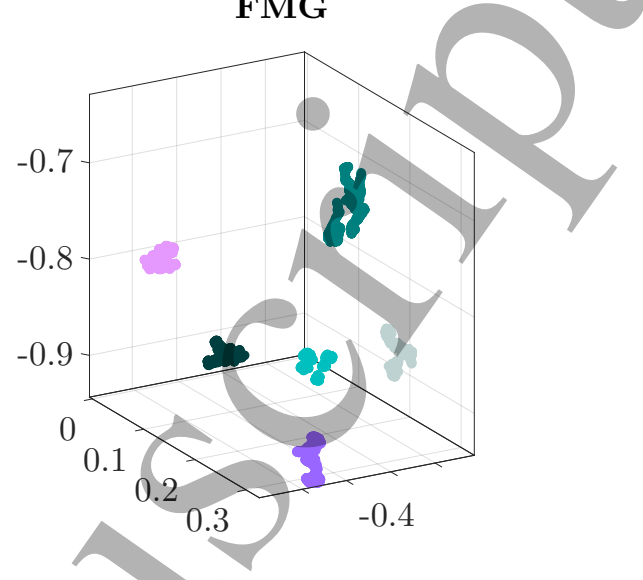

Figure 9: 3D plot of the training data of the median subject (left sEMG, right FMG). LDA was used to reduce the dimensionality to 3. (Rotating version in supplementary material)

The three graphs in Figure 8 further emphasise this behaviour. While at level 1.00 we can see an almost even distribution of different ML configurations, for the lower levels it becomes more and more clustered. We can see that for level 0.33 configurations FMG and stacked are located more to the left at high numbers of successfully accomplished tasks, while the only sEMG configuration can be found on the very right at lower number of successful tasks. The ensemble learning is situated more in the middle. The behaviour at level 0.67 appears to be in between the one at level 0.33 and level 1.00. We speculate that action interference, present whenever sEMG is part of the input space, can "deceive" both mixed approaches, therefore lowering the potentially better perforance obtained by FMG.

\subsection{Conclusions and future work}

FMG achieved a more robust myocontrol than sEMG in our experiment, and could better generalise to activation levels which were not present in the training set. FMG qualifies than once more as a viable and interesting replacement to sEMG in myocontrol, although more experiments/are required, particularly as far as the embedding of FMG sensors in a socket is concerned [4]. The main conclusion we draw from this study is that to achieve a robust myocontrol that is capable to generalise to untrained data we need more than just sEMG sensors. Here, we have shown that the addition of FMG sensors leads to significant improvements, particularly for fine and precise manipulation. We can see, particularly in Figure 5, that to truly achieve proportionality, that means reliable-control along the full spectrum of activations, we can not only rely on sEMG sensor, but need additional information.

We were able to identify the action interference of two DOF of the wrist to be the source of failure at low levels of activation, when using sEMG sensors. This is not the first time we encountered this issue and we already launched investigation to 
find a solution [25]. However, for FMG this interference does not seem to be present. The separability is preserved across the full range of activation and therefore allows fine and precise manipulation. As a general way of eliminating action interference from myocontrol, we envision that one or more quality indexes could be devised, leading to the possibility of ruling out interference even before it would actually happen [25], by increasing or changing the sensor array and/or by extracting different features from the signals.

As a further remark, our comparison shows no advantage of mixing sEMG information with FMG over only using FMG information. This is a very interesting finding. As a last remark, note that this investigation was performed in a seated position without large scale motion of the arm and/or subject and without external load on the hand/prosthesis. These conditions could influence the performance, and lifting this assumption is subject to future investigations.

\section{Acknowledgments}

This work was partially supported by the German Research Society project Tacthand: improving control of prosthetic hands using tactile sensors and realistic machine learning (DFG Sachbeihilfe CA-1389/1-1, http://gepris.dfg.de/gepris/projekt/ 272314643? language $=$ en).

\section{References}

[1] C. Ahmadizadeh, L. K. Merhi, B. Pousett, S. Sangha, and C. Menon. Toward intuitive prosthetic control: Solving common issues using force myography, surface electromyography, and pattern recognition in a pilot case study. IEEE Robotics Automation Magazine, 24(4):102-111, Dec 2017.

[2] P. Beckerle, C. Castellini, and B. Lenggenhager. Robotic interfaces for cognitive psychology and embodiment research: a reselarch roadmap. Wiley Interdisciplinary Reviews - Cognitive Science, page e1486, 2018.

[3] C. Castellini, P. Artemiadis, M. Wininger, A. Ajoudani, M. Alimusaj, A. Bicchi, B. Caputo, W. Craelius, S. Dosen, K. Englehart, D. Farina, A. Gijsberts, S. Godfrey, L. Hargrove, M. Ison, T. Kuiken, M. Markovic, P. Pilarski, R. Rupp, and E. Scheme. Proceedings of the first workshop on peripheral machine interfaces: going beyond traditional surface electromyography. Frontiers in Neurorobotics, 8(22), 2014.

[4] E. Cho, R. Chen, L.-K. Merhi, Z. Xiao, B. Pousett, and C. Menon. Force myography to control robotic upper extremity prostheses: A feasibility study. Frontiers in Bioengineering and Biotechnology, 4:18, 2016.

[5] M. Connan, E. Ruiz Ramírez, B. Vodermayer, and C. Castellini. Assessment of a wearable forceand electromyography device and comparison of the related signals for myocontrol. Frontiers in Neurorobotics, 10(17), 2016.

[6] F. Cordella, A. L. Ciancio, R. Sacchetti, A. Davalli, A. G. Cutti, E. Guglielmelli, and L. Zollo. Literature review on needs of upper limb prosthesis users. Frontiers in Neuroscience, 10:209, 2016. 
Online combination of FMG and sEMG

[7] T. G. Dietterich. Ensemble learning. The handbook of brain theory and neural networks, 2:110$125,2002$.

[8] S. M. Engdahl, B. P. Christie, B. Kelly, A. Davis, C. A. Chestek, and D. H. Gates. Surveying the interest of individuals with upper limb loss in novel prosthetic control techniques. Journal of NeuroEngineering and Rehabilitation, 12(1):53, 2015.

[9] Y. Fang, N. Hettiarachchi, D. Zhou, and H. Liu. Multi-modal sensing techniques for interfacing hand prostheses: A review. IEEE Sensors Journal, 15(11):6065-6076, Nov 2015.

[10] A. Field, J. Miles, and Z. Field. Discovering statistics using R. Sage publications, 2012.

[11] F. R. Finley and R. W. Wirta. Myocoder studies of multiple myopotential response. Archives of Physical Medicine and Rehabilitation, 48(11):598-601, 1967.

[12] A. Fougner, Ø. Stavdahl, P. J. Kyberd, Y. G. Losier, and P. A. Parker. Control of upper limb prostheses: Terminology and proportional myoelectric control - a review. IEEE Trans Neur Syst Rehab Eng, 20(5):663-677, 2012.

[13] A. Gijsberts, R. Bohra, D. Sierra González, A. Werner, M. Nowak, B./Caputo, M. Roa, and C. Castellini. Stable myoelectric control of a hand prosthesis using non-linear incremental learning. Frontiers in Neurorobotics, 8:8, 2014.

[14] N. Jiang, I. Vujaklija, H. Rehbaum, B. Graimann, and D. Farina. Is accurate mapping of emg signals on kinematics needed for precise online myoelectric control? IEEE Transactions on Neural Systems and Rehabilitation Engineering, 22(3):549-558, May 2014.

[15] J. Lobo-Prat, P. N. Kooren, A. H. Stienen, J.L. Herder, B. F. Koopman, and P. H. Veltink. Noninvasive control interfaces for intention detection in active movement-assistive devices. Journal of NeuroEngineering and Rehabilitation, 11(1):168, 2014.

[16] R. Merletti, A. Botter, C. Cescon, M. Minetto, and T. Vieira. Advances in surface EMG: Recent progress in clinical research applications. Critical reviews in biomedical engineering, 38(4):347$379,2011$.

[17] R. Merletti, A. Botter, A. Troiano, E. Merlo, and M. Minetto. Technology and instrumentation for detection and conditioning of the surface electromyographic signal: State of the art. Clinical Biomechanics, 24:122-134, 2009.

[18] M. Nowak, B. Aretz, and C. Castellini. Wrist and grasp myocontrol: Online validation in a goalreaching task. In 2016 25th IEEE International Symposium on Robot and Human Interactive Communication (RO-MAN), pages 132-137, Aug 2016.

[19] M. Nowak and C. Castellini. The let procedure for prosthetic myocontrol: Towards multi-dof control using single-dof activations. PLOS ONE, 11(9):1-20, 092016.

[20] M. Nowak, T. Eiband, and C. Castellini. Multi-modal myocontrol: Testing combined force and electromyography. In Proceedings of ICORR - International Conference on Rehabilitation Robotics, 2017.

[21] M. Ortiz-Catalan, F. Rouhani, R. Brånemark, and B. Håkansson. Offline accuracy: A potentially misleading metric in myoelectric pattern recognition for prosthetic control. In 2015 37th Annual International Conference of the IEEE Engineering in Medicine and Biology Society (EMBC), pages 1140-1143, Aug 2015.

[22] G. Patel, M. Nowak, and C. Castellini. Exploiting knowledge composition to improve real-life hand prosthetic control. IEEE Transactions on Neural Systems and Rehabilitation Engineering, 25(7):967-975, July 2017.

[23] B. Peerdeman, D. Boere, H. Witteveen, R. H. in 't Veld, H. Hermens, S. Stramigioli, H. Rietman, P. Veltink, and S. Misra. Myoelectric forearm prostheses: State of the art from a user-centered perspective. Journal of Rehabilitation Research and Development, 48(6):719-738, 2011.

[24] S. L. Phillips and W. Craelius. Residual kinetic imaging: A versatile interface for prosthetic control. Robotica, 23(3):277-282, May 2005.

[25] L. Rauh. Optimizing myocontrol for daily-life activities. Technical report, Technische Universität München, September 2017.

[26] V. Ravindra and C. Castellini. A comparative analysis of three non-invasive human-machine 
1

2

3

4

5

6

7

8

9

Online combination of FMG and sEMG

interfaces for the disabled. Frontiers in Neurorobotics, 8(24), 2014.

[27] D. Sierra González and C. Castellini. A realistic implementation of ultrasound imaging as a human-machine interface for upper-limb amputees. Frontiers in Neurorobotics, 7(17), 2013.

[28] A. M. Simon, L. J. Hargrove, B. A. Lock, and T. A. Kuiken. Target achievement control test: Evaluating real-time myoelectric pattern recognition control of multifunctional upper-limb prostheses. Journal of Rehabilitation Research \& Development (JRRD), 48(6):619-628, 2011.

[29] I. Strazzulla, M. Nowak, M. Controzzi, C. Cipriani, and C. Castellini. Online bimanual manipulation using surface electromyography and incremental learning. IEEE Transactions on Neural Systems and Rehabilitation Engineering, 25(3):227-234, March 2017.

[30] I. Vujaklija, A. D. Roche, T. Hasenoehrl, A. Sturma, S. Amsuess, D. Farina, and O. C. Aszmann. Translating research on myoelectric control into clinics are the performance assessment methods adequate? Frontiers in Neurorobotics, 11:7, 2017.

[31] M. Wininger, N. Kim, and W. Craelius. Pressure signature of forearm as predictor of grip force. Journal of Rehabilitation Research and Development, 45(6):883-892, 2008.

[32] D. Yungher, M. Wininger, W. Baar, W. Craelius, and A. Threlkeld. Surface muscle pressure as a means of active and passive behavior of muscles during gait. Medical Engineering $\&$ Physics, $33: 464-471,2011$. 